\title{
Coordination Always Occurs in a Two-Strategy Pure-Coordination Logit Game on Scale-Free Networks*
}

\author{
Tomohiko Konno \\ Institute of Advanced Study, Waseda University, Tokyo, Japan \\ Email: tomo.konno@aoni.waseda.jp
}

Received 22 July 2015; accepted 21 August 2015; published 24 August 2015

Copyright (C) 2015 by author and Scientific Research Publishing Inc.

This work is licensed under the Creative Commons Attribution International License (CC BY).

http://creativecommons.org/licenses/by/4.0/

cC) (7) Open Access

\begin{abstract}
We show that coordination always occurs in scale-free networks by social local interactions regardless of the values of parameters, while it occurs in regular networks if and only if the number of links times a payoff parameter exceeds the threshold. Scale-free networks are ubiquitous in the reality. We study a two-strategy pure coordination game on networks that indicate who plays with whom. A player chooses a strategy by Logit choice and the strategies are dynamically updated. Stable steady states are investigated.
\end{abstract}

\section{Keywords}

Games on Networks, Scale-Free Networks, Coordination Games, Local Social Interaction, Strategy Diffusion, Network Heterogeneity

\section{Introduction}

We study a two-strategy pure coordination game on networks that indicate who plays with whom. A player follows logit choice in the games. We show that one strategy always prevails by social local interactions regardless of the values of parameters in scale-free networks, while it prevails if and only if the condition is satisfied with regular networks, which is the number of links times payoff parameter exceeds the threshold. There are a lot of situations in which people derive benefits from choosing the same action as neighbors' ones. We do not interact nor derive benefits directly from all the other people but our neighbors, which is a social network. We study how social networks affect coordination phenomenon. For this purpose, we study a pure coordination game in networks. We show how the heterogeneity in degree distribution affects the cooperation

*This research was partly supported by a grant-in-aid from the Seimeikai Foundation. 
phenomena because real networks are typically heterogeneous. We compare the outcomes of regular networks with those of scale-free networks since regular networks are representative of homogenous networks and scale-free networks are representative of heterogeneous networks. Studying a model in scale-free networks reveals how network heterogeneity affects the outcome. A model in a scale-free network is realistic and significant because many real social networks are scale-free at least in tail distribution. A scale-freeness in tail distribution determines the outcome of a model on a network.

A regular network is a network where all the vertices have the same degree that is the number of links $\xi$. A scale-free network is a network where degree distribution follows $P(\xi) \sim \xi^{-\gamma}$. They are illustrated in Figure 1 and Figure 2. Figure 3 illustrates a degree distribution in a logarithmic plot. We consider scale-free networks with $2<\gamma \leq 3$ since most real scale-free networks are this type. Also the network size is infinity and there is no degree correlation in the present paper.

It is recently found that many social networks such as inter-firm transactions are not alike regular networks rather they are scale-free networks. For example, [1] studies the network of inter-firm transactions in Japan, which is a scale-free and hierarchical network. It is known that an underlying network structure changes an outcome of a model. The network heterogeneity affects an outcome and, in particular, scale-free networks lead to drastic changes because scale-free networks have great network heterogeneity.

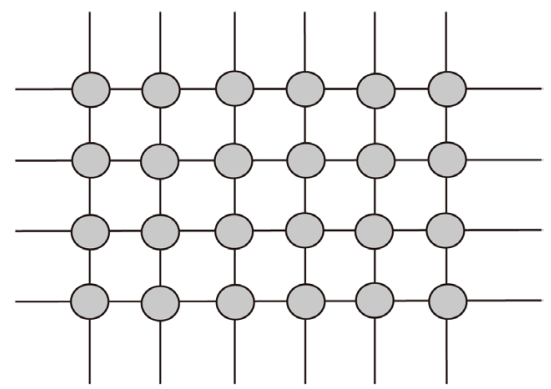

Figure 1. Regular network.

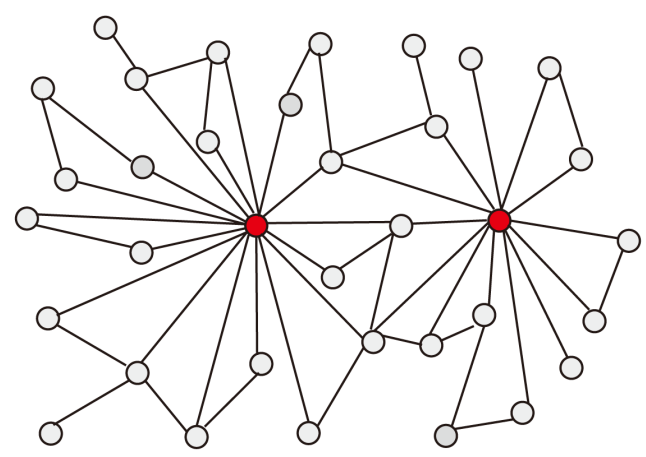

Figure 2. Scale-free network.

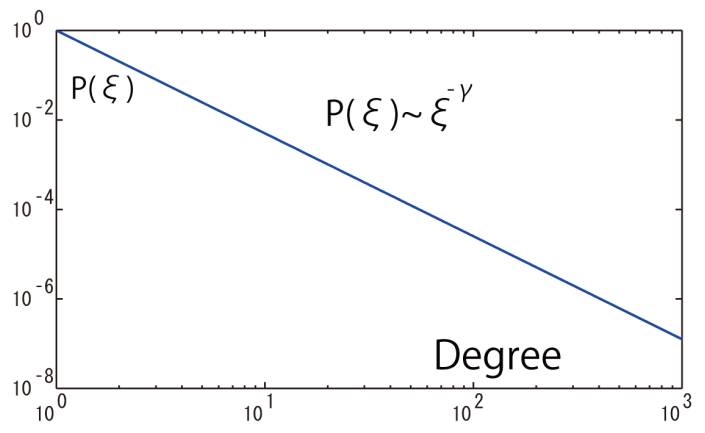

Figure 3. Degree distribution in log-log plot. 


\section{The Model}

There are two strategies, $A$ and $B$ in a coordination game. The payoff matrix is given by

A

B

$$
\begin{array}{cc}
A(2 a, 2 a) & (0,0) \\
B\left(\begin{array}{cc}
(0,0) & (2 a, 2 a)
\end{array}\right) .
\end{array}
$$

If one derives payoff from choosing the same strategy as neighbors', it is called neighborhood effect. No extra payoff is derived by taking either strategy. We consider such a game in order to investigate how the neighborhood effect is strengthened by the network. No strategy is risk dominant and the payoff is symmetric in the game, because the purpose of the present paper is to investigate "pure” neighborhood effect. The case with risk dominance will be studied by our other paper.

A game with this payoff matrix has applications in reality; some examples are provided as follows. Does a player choose which of Social Network Service? PC or Macintosh? Which programming language? In these examples, payoffs arise if one chooses the same strategy as others'. Evidently, there are many kinds of goods with such neighborhood effect. This model can describes the phenomena regarding fashion by focusing on the argument that people tend to follow other people.

Let $s_{i}$ denote the strategy of player $i$ and $u\left(s_{i}, s_{j}\right)$ denote the payoff of player $i$ from the game with player $j$. Because this is a two-strategy coordination game, we assume that $s_{i}$ takes either +1 or -1 without loss of generality, each corresponds to strategy $A$ and $s=-1$ to $B$ respectively. The payoff function $u\left(s_{i}, s_{j}\right)$ is given by

$$
u\left(s_{i}, s_{j}\right)=a s_{i} \cdot s_{j}+a .
$$

A player plays the games with multiple players. The network indicates who plays the games with whom. A player is set on a vertex. Players play games only with players on adjacent vertices and extract payoff from each game. An example is illustrated in Figure 4. This is a standard for games on networks. We derive an average payoff over social interactions in some cases. On the other hand, we derive payoff from each social interaction in some cases. We are going to study such games where the payoff increases as social interactions increases.

Let the notation $j \in \partial i$ denote all the players adjacent to player $i$. The payoff of the player $i$ is given by

$$
u\left(s_{i} ;\left\{s_{j}\right\}_{j \in \partial i}\right)=\sum_{j \in \partial i}\left(a s_{i} \cdot s_{j}+a\right) .
$$

Let $u\left(s_{i}\right)$ denote $u\left(s_{i} ;\left\{s_{j}\right\}_{j \in \partial i}\right)$ in short. The game is dynamic. A randomly chosen player updates the strategy in each time step. The chosen player knows the strategies of adjacent players. We assume that player $i$ chooses the strategy with the following Logit probability:

$$
\operatorname{Pr}\left(s_{i} ;\left\{s_{j}\right\}_{j \in \partial i}\right)=\frac{\exp \left[\lambda u\left(s_{i} ;\left\{s_{j}\right\}_{j \in \partial i}\right)\right]}{\sum_{s_{i}} \exp \left[\lambda u\left(s_{i} ;\left\{s_{j}\right\}_{j \in \partial i}\right)\right]},
$$

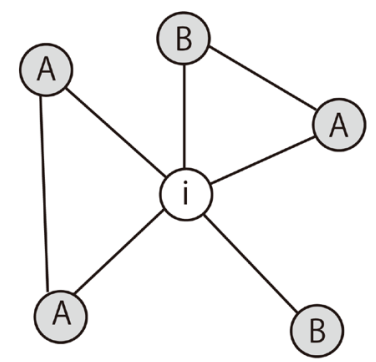

Figure 4. If the strategy of player $i$ is $A$, then the payoff of player $i$ is $6 a$. If the strategy is $B$, the payoff is $4 a$. 
where $\lambda$ is the rationality parameter. After enough number of time steps, the distribution of each strategy becomes stable. We call such state a stable state and we will focus on it. We will see that the probability distribution of strategy in the stable state is independent from initial state. This process can be seen as a diffusion of strategies through local interactions. Diffusion processes on social networks were found in the books by [2]-[4]. Contagion processes on networks were studied by [5]-[9]. Such a functional form as in (4) has been used by several well-known papers, for example [10]-[13].

\section{The Model in a Regular Network}

The analysis of the model in a regular network is discussed in detail in Appendix. If both of the probabilities of choosing strategies $A$ and $B$ are the same, we say that neither strategy prevails. On the other hand, if a probability of either strategy is larger than the other, we define one strategy prevails. To conclude, in regular networks with degree $\xi$.

- If $a \lambda \xi \leq 1$, neither strategy prevails.

- If $a \lambda \xi>1$, one strategy prevails by the neighborhood effect.

A strategy with larger initial probability prevails in the steady state if the condition $a \lambda \xi>1$ is satisfied. This result is yet known. We mention here to compare with that of scale-free networks.

\section{The Model in a Scale-Free Network}

We will show that unlike regular networks, one strategy always prevails regardless of the values of parameters in scale-free networks. In a scale-free network, people tend to choose the same strategy by the neighborhood effect. It also holds true for a heterogeneous network.

\subsection{Mean Field Approximation}

We use the mean-field approximation for heterogeneous networks to solve the model in scale-free networks. The mean-field approximation is developed in our previous paper [14] to the best of our knowledge.

\section{2. $\left\langle\xi_{\mathrm{nn}}\right\rangle$ Network}

Let $\langle\xi\rangle$ denote the mean degree and let $\left\langle\xi_{\text {nn }}\right\rangle$ denote the mean degree of nearest neighbors. Let $\langle\bullet\rangle$ denote $E(\bullet)$. First, we solve for the mean strategy of the player $i$ with degree $\left\langle\xi_{\text {nn }}\right\rangle$. All the adjacent players of the player $i$ also have degree $\left\langle\xi_{\mathrm{nn}}\right\rangle$ in the mean-field approximation. In mean-field approximation, the strategies of adjacent players, $s_{j}\left(\left\langle\xi_{\mathrm{nn}}\right\rangle\right)$ such that $j \in \partial i$, are replaced by the mean strategy of such players, $\left\langle s\left(\left\langle\xi_{\mathrm{nn}}\right\rangle\right)\right\rangle$. Then, the mean of the strategy of players denoted by $i$ that have $\left\langle\xi_{\mathrm{nn}}\right\rangle$ degree is given by

$$
\left\langle s_{i}\left(\left\langle\xi_{\mathrm{nn}}\right\rangle\right)\right\rangle=\frac{\sum_{s_{i}= \pm 1} s_{i} \exp \left[\lambda\left\langle\xi_{\mathrm{nn}}\right\rangle a s_{i} \cdot\left\langle s\left(\left\langle\xi_{\mathrm{nn}}\right\rangle\right)\right\rangle\right]}{\sum_{s_{i}= \pm 1} \exp \left[\lambda\left\langle\xi_{\mathrm{nn}}\right\rangle a s_{i} \cdot\left\langle s\left(\left\langle\xi_{\mathrm{nn}}\right\rangle\right)\right\rangle\right]}=\tanh \left(a \lambda\left\langle\xi_{\mathrm{nn}}\right\rangle s_{\mathrm{nn}}\right) .
$$

where $s_{\mathrm{nn}} \equiv\left\langle s\left(\left\langle\xi_{\mathrm{nn}}\right\rangle\right)\right\rangle$. We have the self-consistency condition that the mean $\left\langle s_{i}\left(\left\langle\xi_{\mathrm{nn}}\right\rangle\right)\right\rangle$ indeed equals to $s_{\mathrm{nn}}$. From the self-consistency condition, Equation (5) is simplified to

$$
s_{\mathrm{nn}}=\tanh \left(a \lambda\left\langle\xi_{\mathrm{nn}}\right\rangle s_{\mathrm{nn}}\right) .
$$

We solve Equation (6) to obtain the mean strategy of players with degree $\left\langle\xi_{\mathrm{nn}}\right\rangle$. Since $\left\langle\xi_{\mathrm{nn}}\right\rangle \rightarrow \infty, s_{\mathrm{nn}}= \pm 1$ holds.

We explained the neighborhood effect problem of players with degree $\left\langle\xi_{\mathrm{nn}}\right\rangle$, we then proceed to the problem of players with arbitrary degree $\xi$. 


\subsection{A Strategy of Player with Degree $\xi$}

Because in mean-field approximation, a vertex with arbitrary degree $\xi$ is surrounded by vertices with degree $\left\langle\xi_{\mathrm{nn}}\right\rangle$, we replace all the strategies taken by adjacent players with $s_{j}\left(\left\langle\xi_{\mathrm{nn}}\right\rangle\right)$. Thus, the average of strategy $s_{i}(\xi)$ is given by

$$
\left\langle s_{i}(\xi)\right\rangle=\sum_{s_{i}= \pm 1} s_{i} \operatorname{Pr}\left(s_{i}\right)=\frac{\sum_{s_{i}= \pm 1} s_{i} \exp \left(\lambda \sum_{j \in \partial i} a s_{i} \cdot s_{j}\left(\left\langle\xi_{\mathrm{nn}}\right\rangle\right)\right)}{\sum_{s_{i}= \pm 1} \exp \left(\lambda \sum_{j \in \partial i} a s_{i} \cdot s_{j}\left(\left\langle\xi_{\mathrm{nn}}\right\rangle\right)\right)} .
$$

We replace all of the $s_{j}\left(\left\langle\xi_{\mathrm{nn}}\right\rangle\right)$ with the mean strategy of the nearest neighbors $s_{\mathrm{nn}} \equiv\left\langle s\left(\left\langle\xi_{\mathrm{nn}}\right\rangle\right)\right\rangle$ in the mean-field approximation. Then, Equation (7) becomes

$$
\left\langle s_{i}(\xi)\right\rangle=\frac{\sum_{s_{i}= \pm 1} s_{i} \exp \left[\lambda \xi a s_{i} \cdot\left\langle s\left(\left\langle\xi_{\mathrm{nn}}\right\rangle\right)\right\rangle\right]}{\sum_{s_{i}= \pm 1} \exp \left[\lambda \xi a s_{i} \cdot\left\langle s\left(\left\langle\xi_{\mathrm{nn}}\right\rangle\right)\right\rangle\right]}=\tanh \left(a \lambda \xi s_{\mathrm{nn}}\right) .
$$

The mean strategy of players with $\xi$ degree is given by

$$
\langle s(\xi)\rangle=\tanh \left(a \lambda \xi s_{\mathrm{nn}}\right) .
$$

Because we already obtained $s_{\mathrm{nn}}=1$, we have $\langle s(\xi)\rangle \neq 0 \forall \xi$. This indicates that in scale-free networks one strategy always prevails and people tend to choose the same strategy regardless of the values of parameters. The strategy with larger initial probability prevails in the steady state. This is the first main result of our paper.

Proposition 1. In the scale-free networks, one strategy always prevails regardless of the values of parameters $a, \lambda$, and $\langle\xi\rangle$, whereas in regular networks, a strategy prevails if and only if the condition a $\lambda \xi>1$ is satisfied. This proposition is within mean-field approximation.

We confirm the proposition by numerical simulations in Section 4.5.

\subsection{Network Heterogeneity}

The network heterogeneity and the mean degree of nearest neighbors are proportional. Therefor, the more heterogeneous a network is, the more likely one strategy prevails.

\subsection{Numerical Simulations}

We will confirm Proposition 1 by numerical simulations. The coordination games are done on regular network and on scale-free network. The mean degree $\langle\xi\rangle=10$, the network size $N=10000$, and $\lambda=0.1$ for both networks. We constructed a scale-free network with $\gamma=2.1$ by BA network formation ([15] [16]). In the beginning, A and B players are equally set randomly. After 50,000 times updates of the games, the game is over. The results are illustrated in Figure 5. In regular network, $|E(s)|$ is almost 0 if $a$ is less than 1 and it is non zero if $a$ is larger than 1 , where the threshold is $a \lambda \xi_{\text {regular }}=1$. The $|E(s)|$ is not 0 in the scale-free network. The numerical simulation confirmed Proposition 1.

\subsection{Intuition: Why Does One Strategy Always Prevail Regardless of the Values of Parameters in Scale-Free Networks?}

Because players on hub vertices are linked to numerous players, the difference between choosing the best strategy and otherwise is huge. One strategy always prevails in hub players es. Most vertices linked to normal vertices, which does not have big degree, are hubs. Because hub players choose the same strategy yet, players on normal vertices choose the same strategy as well. Therefore, one strategy always prevails in scale-free networks. 


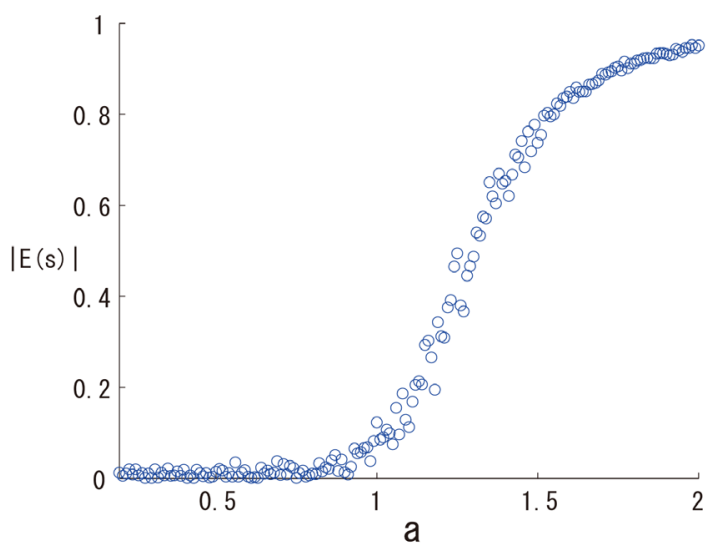

(a)

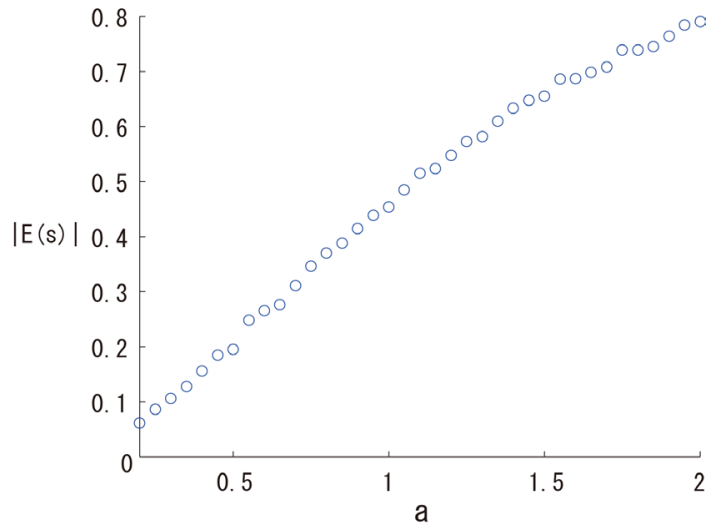

(b)

Figure 5. The $x$ axis indicates the payoff parameter $0.2 \leq a \leq 2$ in the game and the $y$ axis indicates the absolute value of expected strategy $|E(s)|$. (a) Regular Network; (b) Scale-free Network.

\section{Concluding Remarks}

We studied a coordination game in networks. We studied scale-free networks since many real networks were scale-free ones or heterogeneous ones. In regular networks, one strategy prevailed if and only if the condition was satisfied, whereas one strategy always prevailed regardless of the values of parameters in scale-free networks. This suggested that people tended to choose the same strategy in scale-free networks.

\section{References}

[1] Konno, T. (2009) Network Structure of Japanese rms. Scale-Free, Hierarchy, and Degree Correlation: Analysis from 800,000 rms. Economics.

[2] Vega-Redondo, F. (2007) Complex Social Networks. Cambridge Univerity Press, Cambridge. http://dx.doi.org/10.1017/CBO9780511804052

[3] Goyal, S. (2007) Connections: An Introduction to the Economics of Networks. Princeton University Press, Princeton.

[4] Jackson, M. (2008) Social and Economic Networks. Princeton University Press, Princeton.

[5] Ellison, G. (1993) Learning, Local Interaction, and Coordination. Econometrica, 61, 1047-1071.

[6] Morris, S. (2000) Contagion. The Review of Economic Studies, 67, 57-78. http://dx.doi.org/10.1111/1467-937X.00121

[7] Alos-Ferrer, C. and Weidenholzer, S. (2008) Contagion and Efficiency. Journal of Economic Theory, 143, 251-274.

[8] Lopez-Pintado, D. (2006) Contagion and Coordination in Random Networks. International Journal of Game Theory, 34, 371-381.

[9] Galeotti, A., Goyal, S., Jackson, M., Vega-Redondo, F. and Yariv, L. (2010) Network Games. Review of Economic Studies, 77, 218-244.

[10] Blume, L. (1993) The Statistical Mechanics of Strategic Interaction. Games and Economic Behavior, 5, 387-424.

[11] Arthur, W., Durlauf, S. and Lane, D. (1997) The Economy as an Evolving Complex System II. Addison-Wesley, Reading.

[12] Young, H. (2001) Individual Strategy and Social Structure: An Evolutionary Theory of Institutions. Princeton University, Princeton.

[13] Brock, W. and Durlauf, S. (2001) Discrete Choice with Social Interactions. The Review of Economic Studies, 68, 235260.

[14] Konno, T. (2011) A Condition for Cooperation in a Game on Complex Networks. Journal of Theoretical Biology, 269 224-233. http://dx.doi.org/10.1016/j.jtbi.2010.10.033

[15] Barabasi, A. and Albert, R. (1999) Emergence of Scaling in Random Networks. Science, 286, 509-512. http://dx.doi.org/10.1126/science.286.5439.509

[16] Dorogovtsev, S., Mendes, J. and Samukhin, A. (2000) Structure of Growing Networks with Preferential Linking. Physical Review Letters, 85, 4633-4636. 
[17] Baxter, R. (1982) Exactly Solved Models in Statistical Mechanics. Academic Press, London.

[18] Kubo, R. (1965) Statistical Mechanics: An Advanced Course with Problems and Solutions. North-Holland Publishing Company, Haarlem.

[19] Greiner, W., Neise, L. and Stocker, H. (1995) Thermodynamics and Statistical Mechanics. Springer, New York. http://dx.doi.org/10.1007/978-1-4612-0827-3

[20] Ising, E. (1925) Beitrag zur theorie des ferromagnetismus. Zeitschrift fur Physik A Hadrons and Nuclei, 31, $253-258$. 


\section{Appendix A. Analysis of the Model in Regular Network}

We present the result in a regular network that a strategy prevails by the neighborhood effect if and only if the condition $a \lambda \xi>1$ is satisfied. Although the result might be yet known, we will discuss it in detail because it will act as a useful reference to those who are not familiar with this topic and because we will compare the result with that in a scale-free network. The discussion including [13], this paper, and other works employ the method developed to solve the phase transition of the Ising model. Please see references such as [17]-[20].

We let $\langle x\rangle$ denote the expected value of a random variable $x:\langle x\rangle \equiv E(x)$. Because expected values appear frequently, it would be confusing to express the expectation value as $E(x)$. The probability that a player $i$ chooses strategy $s_{i}$ is given by

$$
\begin{aligned}
\operatorname{Pr}\left(s_{i}\right)= & \operatorname{Pr}\left(s_{i} ;\left\{s_{j}\right\}_{j \in \delta i}\right)=\frac{\exp \left[\lambda u\left(s_{i} ;\left\{s_{j}\right\}_{j \in \partial i}\right)\right]}{\sum_{s_{i}} \exp \left[\lambda u\left(s_{i} ;\left\{s_{j}\right\}_{j \in \partial i}\right)\right]} \\
= & \frac{\exp \left(\lambda \sum_{j \in \partial i}\left(a s_{i} \cdot s_{j}+a\right)\right)}{\sum_{s_{i}= \pm 1} \exp \left(\lambda \sum_{j \in \partial i}\left(a s_{i} \cdot s_{j}+a\right)\right)}=\frac{\exp \left(\lambda \sum_{j \in \partial i} a s_{i} \cdot s_{j}\right)}{\sum_{s_{i}= \pm 1} \exp \left(\lambda \sum_{j \in \partial i} a s_{i} \cdot s_{j}\right)}
\end{aligned}
$$

The mean of strategy $s_{i}$ is then given by

$$
\left\langle s_{i}\right\rangle=\sum_{s_{i}= \pm 1} s_{i} \operatorname{Pr}\left(s_{i}\right)=\frac{\sum_{s_{i}= \pm 1} s_{i} \exp \left(\lambda \sum_{j \in \partial i} a s_{i} \cdot s_{j}\right)}{\sum_{s_{i}= \pm 1} \exp \left(\lambda \sum_{j \in \partial i} a s_{i} \cdot s_{j}\right)}
$$

We solve the model by mean-field approximation in which the strategies taken by adjacent vertices are replaced by the average value of strategy $\langle s\rangle$. We need to use mean-field approximation for such models. Then, Equation (A.2) turns into

$$
\left\langle s_{i}\right\rangle=\frac{\sum_{s_{i}= \pm 1} s_{i} \exp \left(\lambda \sum_{j \in \partial i} a s_{i} \cdot\langle s\rangle\right)}{\sum_{s_{i}= \pm 1} \exp \left(\lambda \sum_{j \in \partial i} a s_{i} \cdot\langle s\rangle\right)}=\frac{\sum_{s_{i}= \pm 1} s_{i} \exp \left(\lambda \xi a s_{i} \cdot\langle s\rangle\right)}{\sum_{s_{i}= \pm 1} \exp \left(\lambda \xi a s_{i} \cdot\langle s\rangle\right)}=\tanh (a \lambda \xi\langle s\rangle)
$$

where $\tanh (x) \equiv \frac{\mathrm{e}^{x}-\mathrm{e}^{-x}}{\mathrm{e}^{x}+\mathrm{e}^{-x}}$. Furthermore, because $\left\langle s_{i}\right\rangle=\langle s\rangle \forall i$, called the self consistency condition, must be satisfied, Equation (A.3) becomes

$$
\langle s\rangle=\tanh (a \lambda \xi\langle s\rangle)
$$

Thus, the mean of strategy, $\langle s\rangle$, is given by the intersection between the following equations.

$$
\begin{gathered}
y=\langle s\rangle \\
y=\tanh (a \lambda \xi\langle s\rangle)
\end{gathered}
$$

Because

$$
\frac{\mathrm{d}}{\mathrm{d} x} \tanh (x) \geq 0, \quad \frac{\mathrm{d}^{2}}{\mathrm{~d} x^{2}} \tanh (x) \leq 0
$$

there are only two cases that are illustrated in Figure 6 and Figure 7, in which the lines are Equation (A.5) and the curves are Equation (A.6).

We will study two cases one by one. 


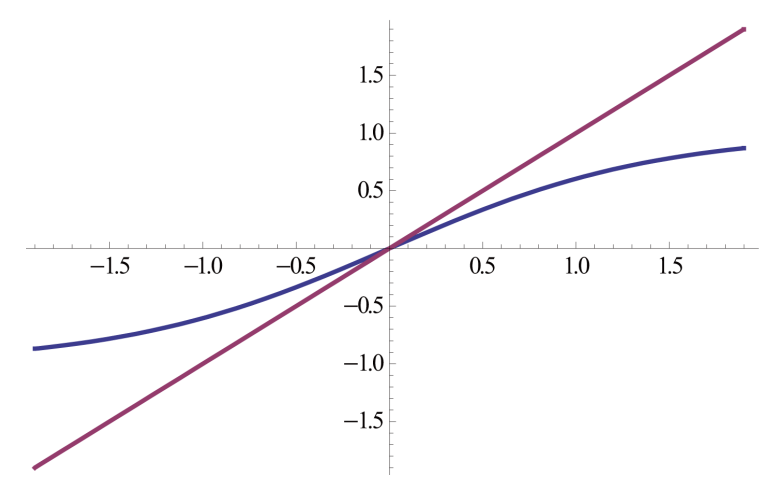

Figure 6. Case A.

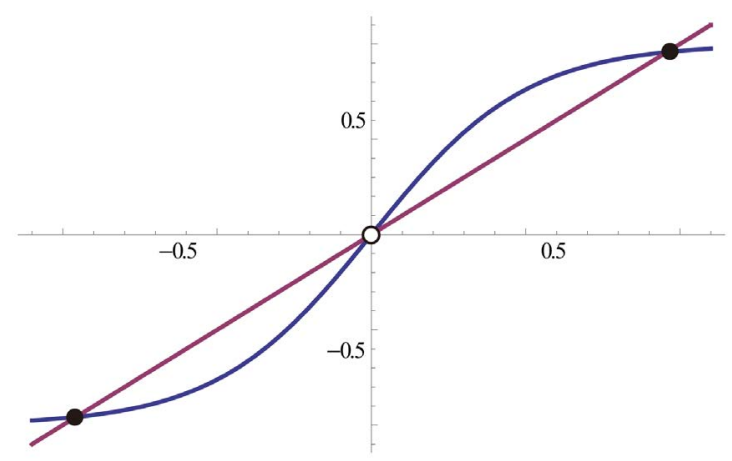

Figure 7. Case B.

\section{Appendix A.1. Case A: $a \lambda \xi \leq 1$}

This case is illustrated in Figure 6. The only fixed point is the origin and it is stable, because the slope of tangent hyperbolic function at the origin is less than 1 . The average of the strategy over all the players in the network is $\langle s\rangle=0$. Because

$$
\left.\frac{\mathrm{d}}{\mathrm{d} x} \tanh (x)\right|_{x=0}=1
$$

Then, $\langle s\rangle=0$ holds if and only if

$$
a \lambda \xi \leq 1
$$

If both strategies are equally likely, $\langle s\rangle \equiv E(s)=+1 \times \frac{1}{2}-1 \times \frac{1}{2}=0$. In contrast, if the frequency of one strategy is larger than the other, $\langle s\rangle=+1 \times p-1 \times(1-p) \neq 0$, where $p \neq \frac{1}{2}$. Therefore, neither strategy prevails if $\langle s\rangle=0$. On the other hand, one strategy prevails if and only if $\langle s\rangle \neq 0$; this is the criterion. Under a strong neighborhood effect, players tend to choose one strategy, and this strategy prevails in the entire network. We show that if and only if $a \lambda \xi>1$, one strategy prevails by the neighborhood effect in a regular network.

\section{Appendix A.2. Case B: $a \lambda \xi>1$}

This case is illustrated in Figure 7. There are three fixed points; however, the origin is an unstable fixed point because $\left.\frac{\mathrm{d}}{\mathrm{d} x} \tanh (x)\right|_{x=0}>1$. The other two fixed points are stable because the derivatives of $\tanh (x)$ w.r.t. $x$ 
at these two points are less than 1. Because the dynamics of $\langle s(t)\rangle$ is given by $\langle s(t+1)\rangle=\tanh (a \lambda \xi\langle s(t)\rangle)$, the stable fixed points are distinguished from the unstable ones. The two stable fixed points exhibit symmetry to the origin; thus, we only need to study the positive fixed points. This case occurs if and only if

$$
a \lambda \xi>1
$$

and the initial value of $\langle s(t=0)\rangle>0$. The negative fixed point realizes if the initial value $\langle s(t=0)\rangle<0$. Therefore, if $a \lambda \xi>1$ then one strategy prevails by the neighborhood effect.

To conclude, in a regular network with degree $\xi$.

- If $a \lambda \xi \leq 1$, neither strategy prevails.

- If $a \lambda \xi>1$, one strategy prevails by the neighborhood effect. 